Paper

UDC 621-503:621-531.4

\title{
Variable Structure Systems Theory Applied to Sub-Time Optimal Position Control with an Invariant Trajectory
}

$\mathrm{By}$

\author{
Okyay Kaynak Fumio Harashima Hideki Hashimoto \\ Non-member Member Member
}

\begin{abstract}
Summary
A microprocessor based sliding mode controller as applied to the position control of a DC servo motor is described. In order to achieve an invariant and at the same time a fast response, the slope of the sliding line is incremented starting with a small initial value. The control law implemented has a switched current feedback term and a switched error velocity term in addition to the normal switched error term. Experimental results are presented showing the invariant nature of the system.
\end{abstract}

\section{Introduction}

In many control applications, the control engineer is required to design a system that will be insensitive to parameter variations and disturbances. This can be a difficult task especially in cases where the parameters of the system vary over a wide range and/or the system is under the influence of a large disturbance. The non-linearities which are almost invariably present in any system make the task even more difficult. The use of high gain coefficients can reduce the effects of parameter variations, disturbances and distortions, but, stability considerations limit the application of this method.

By use of the linear model-following control (LMFC) theory, the designer can shape the error transient response. However, LMFC is again inadequate in the presence of large parameter variations and this has led to the development of adaptive model following control (AMFC) theory.

For the design of an AMFC system, two methods are generally used. One is based on the hyperstability concept $^{(1)}$ and the other on the Lyapunov functions ${ }^{(2)}$, but neither of these methods gives the designer a direct quantitative control over the error transient, only guarantees that the error between the states of the model and the plant goes to zero.

A method of control, called sliding mode control, which has been a subject of extensive theoretical study in the past ${ }^{(3) \sim(6)}$, has recently re-attracted attention and is suggested to enable the designer to prescribe the shape of the transient response ${ }^{(7) \sim(9)}$. In this method,

Okyay Kaynak is with Boğaziç University, Fumio Harashima \& Hideki Hashimoto are with Institute of Industrial Science, University of Tokyo. Manuscript received Jan. 11, 1983, revised Oct. 12, 1983. the representative point of the system is constrained to move (or slide) along a predetermined hyperplane or hyperplanes in state space. In order to achieve such a sliding mode, the control law is required to have a discontinuous nature, i.e. the system structure needs to be changed in time, resulting in a variable structure system (VSS). If the deviations from the sliding plane are small, the motion of the system is completely determined by the chosen plane and consequently the changes in the parameters and the disturbances cannot effect the behavior. The system can therefore be described as a model-following control system. In fact, if the Lyapunov's method is used for AMFC of a single-input single-output system, it is seen that the control law has to be discontinuous, i.e. a VSS ${ }^{(n)}$. The design of a model-following controller for a multiinput system can also be made by the use of VSS theory ${ }^{(8)(10)}$.

In this paper, the design and the implementation of a microprocessor based sliding mede controller for a position control system is described. The controller is easily implemented and it can be shown that ${ }^{(6)}$ the resulting response is close to the desired time optimal trajectory. As a result of some previous studies $^{(11 ;-12)}$, a switched current feedback term and a switched error velocity term are included in the control law in addition to the normal switched error term and a small relay-type term.

In order to achieve both an invariant and a fast response, the slope of the sliding line is adaptively varied as suggested in Ref. (6). Starting with a small initial value, the slope is incremented checking the existence of a sliding mode at every sampling instant. In this way, an invariant response is obtained even in the presence of an appreciably large variation of the system parametrs.

本稿は Section E (Trans. I.E.E. of Japan, Vol, 104, No. 3/4, Mar/Apr, p. 47) 加らの枟载である。 


\section{Description of the system}

The hardware details of the system used for experimental investigations are shown in Fig. 1. A $24 \mathrm{~V}, 50 \mathrm{~W}$ $\mathrm{DC}$ servo motor is driven by a PWM power MOSFET chopper operating at $10 \mathrm{kHz}$. A 10 bit digital shaft encoder is used to sense the output position while a tachogenerator coupled directly to the servo motor provides an analog signal for the output speed. A 10 bit tracking type $\mathrm{A} / \mathrm{D}$ converter converts this into a digital form as required. Another 10 bit $\mathrm{A} / \mathrm{D}$ converter is used to detect the current through an isolation amplifier. The microcomputer used is a Z-80 based system with a $2.5 \mathrm{MHz}$ clock. A gear train is inserted between the servo motor and the output shaft encoder.

Since the tracking type $A / D$ converter have an inherent low pass filter character, any high frequency noise that might be present in the output of the tachogenerator and the current sensor is eliminated.

Fig. 2 shows the equivalent block diagram of the system. The servo motor is modelled as a first order system neglecting the electrical time constant.

The arrangement shown in Fig. 3 was used for the generation of a disturbance, the maximum value of which could be varied by varying the mass $M$ or its distance $L$ from the center of the shaft. Although the disturbance is then a sinusoidal function of the output

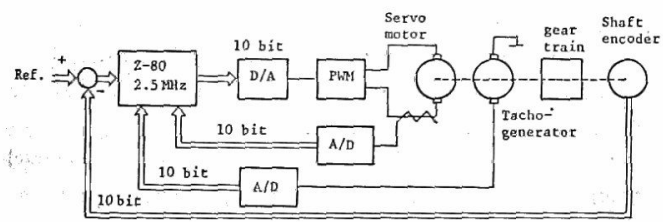

Fig. 1. The hardware details of the system.

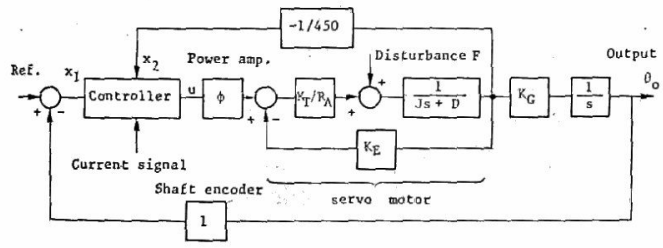

Fig. 2. Block diagram of the system.

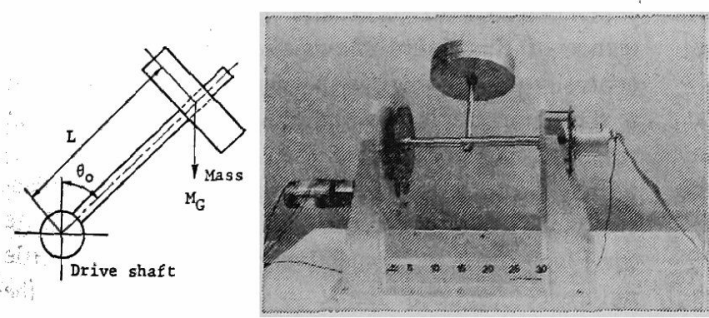

Fig. 3. The mechanical arrangement for the generation of an appreciably large disturbance. position and therefore one of the state variables of the system, the treatment of it as such would unduly complicate the analysis. It was therefore decided to treat it as a constant, taking the maximum value and all the experimental investigations were carried out around the $\pm 90^{\circ}$ output position, which in the worst condition.

The parameters of the system are given in Table 1.

From Fig. 2, the phase variable state representation of the system can be written as follows:

$$
\left[\begin{array}{c}
\dot{x}_{1} \\
\dot{x}_{2}
\end{array}\right]=\left[\begin{array}{rr}
0 & 1 \\
0 & -b
\end{array}\right]\left[\begin{array}{l}
x_{1} \\
x_{2}
\end{array}\right]+\left[\begin{array}{c}
0 \\
-a \phi
\end{array}\right][u]+\left[\begin{array}{l}
0 \\
1
\end{array}\right][f]
$$

$$
\left.\begin{array}{l}
x_{1}: \text { position error } \\
x_{2}: \dot{x}_{1}: \text { error velocity } \\
a=K_{\tau} K_{G} / J R_{A}=1.75 \\
b=\left(K_{T} K_{E}+D R_{A}\right) / J R_{A}=95 \\
f=\left(K_{G} F / J\right) \sin \theta_{0},-1<f<1
\end{array}\right\}
$$

\section{Design and Experimental Investi- gations}

The control implemented is of the form

$$
u=\phi_{1} x_{1}+\psi_{2} x_{2}+\psi_{3} i+k_{f} \text { sgns }
$$

where

$$
\begin{array}{r}
\psi_{1}=\left\{\begin{array}{ll}
\alpha_{1} & \text { if } s x_{1}>0 \\
\beta_{1} & \text { if } s x_{1}<0
\end{array}, \phi_{2}= \begin{cases}\alpha_{2} & \text { if } s x_{2}>0 \\
\beta_{2} & \text { if } s x_{2}<0\end{cases} \right. \\
\psi_{3}=\left\{\begin{array}{ll}
\alpha_{3} & \text { if } s i>0 \\
\beta_{3} & \text { if } s i<0
\end{array}, \text { sgn } s= \begin{cases}+1 & \text { if } s>0 \\
-1 & \text { if } s<0\end{cases} \right. \\
s=x_{2}+c x_{1}, c>0
\end{array}
$$

The first term in Eq. ( 3 ) is the normal proportional. term, the second term relaxes the constraints on the slope $c$ of the sliding line $s=0$ and the third term ensures that the syntem does not come out of the slidiing mode due to the disturbance $f$ without causing intolerable oscillations (chattering) at the origin. The relay type term $k_{f}$ sgn $s$ is like the high frequency "dither" signal frequently used in control systems to overcome the effects of backlash and coulomb frictional forces ${ }^{(13)}$. A detailed discussion on the effects of each of the terms of Eq. ( 3 ) is given in Ref. (12).

The $\phi_{3} i$ term can also be expressed in the form

Table 1. The parameters of the system.

\begin{tabular}{l}
$K_{E}=6.0 \times 10^{-2} \mathrm{~V} \cdot \mathrm{s} / \mathrm{rad}$ \\
$K_{T}=6.0 \times 10^{-2} \mathrm{~N} \cdot \mathrm{m} / \mathrm{A}$ \\
$R_{A}=1.27 \Omega$ \\
$D=2.84 \times 10^{-3} \mathrm{~N} \cdot \mathrm{m} \cdot \mathrm{s} / \mathrm{rad}$ \\
$J=6 \times 10^{-5} \mathrm{~kg} \cdot \mathrm{m}^{2}$ \\
$K_{C}=1 / 450$ \\
$\phi=57$ \\
$L=0.14 \mathrm{~m}$ \\
$M_{G}=9 \mathrm{~kg}$ \\
$F=K_{G} L M_{G} \sin \theta_{0}=27.5 \sin \theta_{0}(\mathrm{~N} \cdot \mathrm{m})$ \\
\hline
\end{tabular}




$$
\psi_{3} i=\left(\alpha_{3}+\beta_{3}\right) i / 2+\left(\alpha_{3}-\beta_{3}\right)|i| \text { sgns } / 2
$$

which, when $\alpha_{3}=-\beta_{3}$, reduces to

$$
\psi_{3} i=\alpha_{3}|i| \operatorname{sgn} s
$$

i.e. it has a similar structure to that of $k_{f}$ with the difference that when the state is forced to zero, this terms also reduces to zero.

The existence of a sliding mode requires that ${ }^{(4)}$

$$
\lim _{s \rightarrow+0} \dot{s} \leq 0 \text { and } \lim _{s \rightarrow-0} \dot{s} \geq 0 \text { or } \lim _{s \rightarrow 0} s \dot{s} \leq 0 .
$$

Neglecting the two relay type components of $\mathrm{Eq}$ (3)

$$
\begin{aligned}
s \dot{s} & =s\left(\dot{x}_{2}+c \dot{x}_{1}\right) \\
& =s\left(-b x_{2}-a \phi \psi_{1} x_{1}-a \phi \psi_{2} x_{2}+c x_{2}\right) \\
& =-a \phi \psi_{1} s x_{1}+\left(c-b-\alpha \phi \psi_{2}\right) s x_{2}
\end{aligned}
$$

is obtained. The inequalities of Eq. ( 7 ) will therefore be satisfied when

$$
a_{1} \geq 0, \beta_{1} \leq 0, a \phi \alpha_{2} \geq c-b \text { and } a \phi \beta_{2} \leq c-d
$$

The slope of the sliding line $c$ should therefore be chosen accordingly, considering the range of the parameters of the system, $a, b$ and $\phi$.

In the derivation of the inequalities of Eq. (9), it is assumed that the control is unrestricted. In the design of a practical system, the fact that the control is limited to a value $|u|<u_{\max }$ should be taken into consideration. This is discussed later in this section.

During the experimental investigations, the phase variable state representation Eq. ( I ) of the system was expressed in discrete form and a number of digital simulations were carried out ${ }^{(12)}$. The following values were finally decided upon.

$$
\left.\begin{array}{l}
\alpha_{1}=1, \beta_{1}=-1 / 2, \alpha_{2}=1 / 8, \beta_{2}=-1, \\
\alpha_{3}=-\beta_{3}=1 / 16 \text { and } k_{f}=4 \text { bits. }
\end{array}\right\}
$$

If the deviations from the sliding line are small, the equation of the motion along the sliding line is the same as that of the sliding line, i.e.

$$
x_{2}+c x_{1}=0
$$

the solution of which is

$$
x_{1}(t)=x_{1}\left(t_{0}\right) \exp \left\{-c\left(t-t_{0}\right)\right\}
$$

In Eq. (12), to is the time of hitting the sliding line and $1 / c$ is the time constant. For a fast response, $c$ has to be as high as possible, but, on the other hand, if $c$ is large, $t_{0}$ will increase. Since the system is invariant only when in a sliding mode, a correspondingly larger part of the trajectory will be sensitive to parameter variations and disturbances.

Fig. 4 shows a typical phase plane trajectory for a constant value of $c$ equal to 16 and Fig. 5 shows the error waveforms for two different conditions. For Fig.5( a ), the supply voltage $V_{s}$ of the chopper is set to $26 \mathrm{~V}$ and the mass $M$ is made zero. For Fig.5(b), the supply voltage is increased to $31.2 \mathrm{~V}$, which means that $\phi$ is increased by $20 \%$ and the full disturbance, i.e. $f=1$, is applied. The test is carried out in such

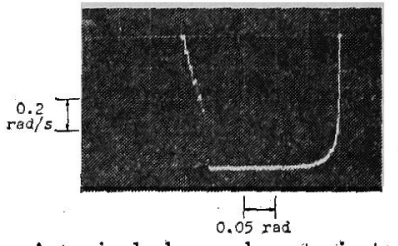

Fig. 4. A typical phase-plane trajectory $(c=16)$.

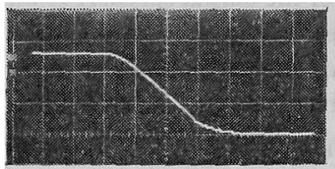

(a) $\quad V_{\mathrm{s}}=26 \mathrm{~V}$ and $f=0$ Ver. scale : 0.1 rad, hor. scale $: 0.1 \mathrm{~s}$

Fig. 5. Error waveforms for $c=16$.

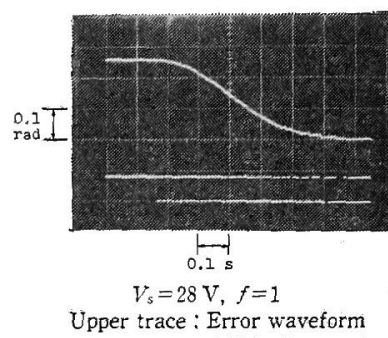

Lower trace: MSB of control

Fig. 6. Waveforms for variable $c$.

a shaft position that the effect of the mass $M$ is additive to the effect of the increase in $V_{s}$, i.e. helping to the rotation of the saft.

Fig. 4 indicates that the aliding line is reached towards the end of the transient trajectory and, as a consequence, the error transients of Fig. 5(a) and Fig. 5 ( b ) are different due to the parameter variation and the disturbance.

A sliding mode throughout the transient response can be ensured if the slope of the sliding line is varied adaptively as is.suggested in Ref.(6), starting with a small initial value and increasing it at each time the existence of a sliding mode is verifield. The upper trace of Fig. 6 shows the error waveform obtained using such an approach for $V_{s}=28 \mathrm{~V}, f=1, c(n+1)$ $=c(n)+0.25, c(0)=0.25$ and $c_{\max }=16$. The lower trance of this figure shows the MSB of the digital control voltage, which is the sign bit. It indicates that a sliding mode is ensured throughout the transient response.

Fig. 7 (a), (b) show the error waveforms for adaptively varied $c$ under the same conditions as those for Fig. 5(a), ( b ) respectively. There is very little difference between the two oscillograms, i.e. the response is invariant in spite of the large change in $\phi$ and the disturbance. 


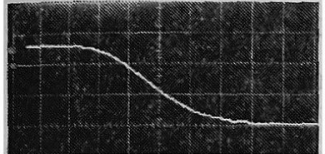

(a) $V_{s}=26 \mathrm{~V}$ and $f=0$ Ver. scale : 0.1 rad, hor. scale : $0.1 \mathrm{~s}$

Fig. 7. Error waveforms for variable $c$.

In the design and the realization of a sliding mode controller, special attention has to be paid to the switching frequency. The higher it is, the less will the deviations be from the ideal sliding line. Furthermore, the devices used to sense the state variables should have high resolution and accuracy. Only then will the motion of the system be almost the same as the determined by the sliding line.

In a digital implementation, the switching frequency will be determined by the sampling period. Utmost attention was therefore paid in the development of the software for the system described. The flowchart of the program is given in Fig. 8. Due to the tracking type A/D converters employed; the digital values of the state variables and the motor current are always available at the input ports. The program is therefore written in a loop form, starting with an input of the variables, calculating $s$, checking whether a transition from one side of the sliding line to the other side has occurred, if so, increasing the value of $c$ for the next cycle and finally calculating the corresponding control voltage. After outputting the digital control voltage, the program returns to the start. A provision is made to reset $c$ to the initial value when zero bit error condition is reached. The maximum value of $c$ is set to 16 .

To facilitate a quick calculation of the control voltage, the coefficients of Eq. (3) were chosen as positive or negative powers of two as indicated in Eq. (10). For the determination of the $c x_{1}$ term of the sliding line, an external 16 bit by 16 bit hardware multiplier is used. With these precautions, the program execution time is kept within $0.5 \mathrm{~ms}$ depending on the outcome of the tests. When the error is zero bits, a shorter routine with a maximum execution time of 0.35 $\mathrm{ms}$ is chosen. A variable sampling time is therefore inherent in the system.

All the discussions presented in this section assume that the control $u$ is unrestricted, which will not be the case in a practical implementation. The control $u$ being restricted to $|u|<u_{\max }$ will, in practice, mean a limited acceleration and deceleration and a limited output speed.

For a simple control law, the regions of the phase plane in which a sliding mode is possible can be derive $d^{\left({ }^{6}\right)}$ but, for a control law involving many terms as implemented in this work, this can prove rather

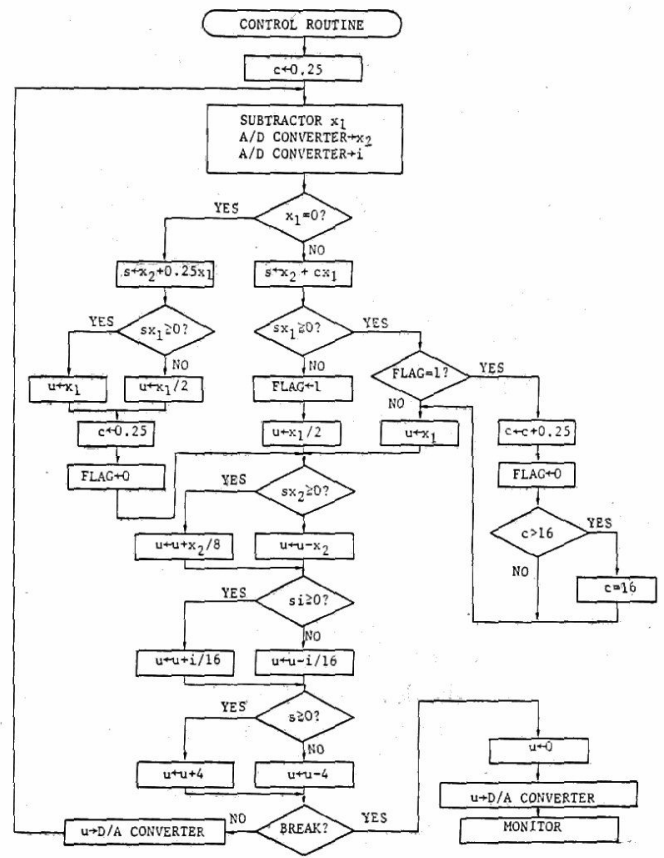

Fig. 8. Flowchart of the program.

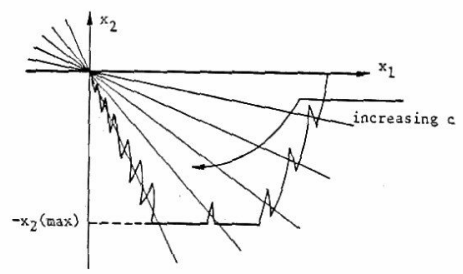

Fig. 9. A typical phase plane trajectory for restricted control.

difficult. However, a quantitative explanation of the effect of the restricted control can be given with reference to Fig. 9.

Since the inequalities of Eq. ( 9 ) are satisfied for the initial value 0.25 of $c$ for any initial position, the phase plane trajectory until the maximum speed is reached will be as shown in Fig. 9 in an exaggerated form. When the maximum speed corresponding to the full control, i.e. the maximum output voltage of the chopper, is reached, the trajectory will be clipped as shown. The next sliding line will however still be hit and the sliding mode will continue. The maximum value of $c$ chosen also satisfies the inequalities of Eq. ( 9 ). When this line is hit, a sliding motion will ensure that the state reaches the origin.

The effect of the restricted control can also be seen 
in Fig. 4. The phase plane trajectory would have been a section of a parabola if $u$ were unrestrícted.

\section{Conclusions}

This paper demonstrates that variable structure systems theory can be used effectively when the invariancy of a system is a requirement. By ensuring that a sliding mode exists throughout the transient response, the system is made insensitive to parameter variations and disturbances. Furthermore, the shape of the transient trajectory can be prescribed by the designer. Although a simple algorithm is used in this work for the definition of the sliding curve, namely, a line with a variable slope, any shape of transient trajetory can be obtained by appropriately defining the sliding curve.

In a digital implementation of a sliding mode controller, the sampling period determines the switching frequency. It must therefore be made as small as possible to keep the deviations from the sliding line to a minimum. The same consideration must be given to the control lag.

The authors would like to acknowledge the fellowship extended to Dr, O. Kaynak by the Matsumae International Foundation of Japan and also the advice and the assistance of Mr. S: Kondo and Mr. H. Inaba.

\section{References}

(1) I. D. Landau: "A survey of mode reference adaptive techniques-Theory and applications", Automatica, 10, 353 (1974)

(2) L. Grayson: "The status of synthesis using Lyapunov's method", ibid, 3,91 (1965)

(3) U. Itkis : Control Systems of Variable Structure (1976) Wiley

(4) V.I. Utkin: "Variable stmucture systems with sliding modes", IEEE Trans. Automatic Control, AC-22, 212 (1977)

(5) B. Drazenovic: "The invariance conditions in variable structure systems", Automatica, 5, 287 (1969)

(6) A.S.1. Zinober: "Adaptive relay control of second-order systems", Itut. J. Control, 21, 81 (1975)

(7) K.-K. D. Young: "Asymptotic stability of model reference systems with variable structure control", IEEE Trans. Automatic Control, AC-22, 279 (1977)

(8) K.-K. D. Young: "Design of variable structure model following control systems", ibid., AC-23, 1079 (1978)

(9) C.A. Klein \& J. J. Maney: "Real-time control of a multiple element mechanical linkage with a microcomputer", IEEE Trans, Indistr. Electronics Control Instrumt, IEC1-26, 227 (1979)

(10) A.S.I. Zinober, O.M.E. EL-Ghezawi \& S.A. Billings: "Multivariable variable-structure adaptive model-following control systems",IEE Proc, 129, Pt. D, 6 (1982)

(11) O. Kaynak, F. Harashima \& M. Okuda : Sliding mode in a position control servo system", Proc. Int. Conf. Elec. Mach., IECM-82, Pt. 1, 137 (1982)

(12) O. Kaynak, F. Harashima \& S. Kondo: "Microprocessor controlled position seryo system with a sliding mode", ETG Conf. Microelectronics in Power Electronics and Electrical Drives, p. 273 (1982)

(13) S. M. Shinners : Control System Design (1966) Wiley 